
\title{
1
}

\section{Health service utilisation amongst urban Aboriginal and Torres Strait Islander children aged younger than 5 years registered with a primary health-care service in South-East Queensland}

\author{
Kerry K Hall (D, , ${ }^{1}$ Anne B Chang, ${ }^{1,2,3}$ Jennie Anderson, ${ }^{4}$ Daniel Arnold, ${ }^{1}$ Michael Otim ${ }^{1,5}$ and Kerry-Ann F O'Grady (D)
}

${ }^{1}$ Centre for Children's Health Research, Institute of Health and Biomedical Innovation, Queensland University of Technology, ${ }^{3}$ Department of Respiratory Medicine, Lady Cilento Children's Hospital, ${ }^{4}$ Caboolture Community Medical, Brisbane, Queensland, ${ }^{2}$ Menzies School of Health Research, Charles Darwin University, Darwin, Northern Territory, Australia and ${ }^{5}$ Department of Health Services Administration, University of Sharjah, Sharjah, United Arab Emirates

Aim: The majority of Australia's Aboriginal and/or Torres Strait Islander children live in urban areas; however, little is known about their health service use. We aimed to describe health service utilisation amongst a cohort of urban Aboriginal and/or Torres Strait Islander children aged $<5$ years.

Methods: We analysed health service utilisation data collected in an ongoing prospective cohort study of children aged $<5$ years registered with an Aboriginal-owned and operated primary health-care service. Enrolled children were followed monthly for 12 months, with data on health service utilisation collected at baseline and at each monthly follow-up. Health service utilisation rates, overall and by service provider and reason for presentation were calculated and reported as incidence rates per 100 child-months with the corresponding $95 \%$ confidence interval (CI).

Results: Between February 2013 and November 2015, 180 children were enrolled, and 1541 child-months of observation were available for analysis. The overall incidence of health service utilisation was 52.5 per 100 child-months $(95 \% \mathrm{Cl}, 48.7-56.5)$; $81 \%$ of encounters were with general practitioners. Presentation rates were the highest for acute respiratory illnesses $(30.7 / 100$ child-months, $95 \% \mathrm{Cl} 27.8-33.9)$.

Conclusions: In this community, acute respiratory illnesses are predominant causes of health service utilisation in young children. The healthcare utilisation profile of these children presents important opportunities for health promotion and intervention.

Key words: Aboriginal and/or Torres Strait Islander; child health; health service utilisation; urban.

\section{What is already known on this topic}

1 Access to appropriate primary health-care services, important for early diagnosis and management, is important for improving the health of Aboriginal and/or Torres Strait Islander children who have a high burden of disease.

\section{What this paper adds}

1 In the cohort examined, the frequent use of health services provides opportunities for education and prevention programmes to be better implemented to address potential health risks.
Correspondence: Kerry K Hall, Centre for Children's Health Research, Institute of Health and Biomedical Innovation, Queensland University of Technology, 62 Graham Street, South Brisbane, Qld. 4101, Australia. Fax: +XXX; email: kerryhall0110@gmail.com

Conflict of interest: J Anderson is the Director of Caboolture Community Medical. J Anderson had no role in the recruitment, consent and followup of study children. KK Hall, AB Chang, D Arnold and KF O'Grady have no conflicts of interest to declare.

Accepted for publication 18 October 2017 addictions, ${ }^{3,4}$ it is known that the accessibility of good primary health care is important. ${ }^{5}$

Primary health-care services that address the historical, social, cultural and economic determinants of health in Aboriginal and/or Torres Strait Islander people, and are culturally acceptable to the community, are important. ${ }^{6}$ There is evidence to suggest that Aboriginal and/or Torres Strait Islander people are less likely to access health services and encounter more barriers in the use of health services. ${ }^{7,8}$ These barriers lead to delays in diagnosis and treatment or lack of exposure to health education and promotion programmes. International studies have shown that failure to access appropriate child health services and early interventions can result in poor health comes in childhood and negative health outcomes in adulthood. ${ }^{9-11}$

There were an estimated 200245 Aboriginal and Torres Strait Islander children in Australia in 2011, comprising 4.9\% of the total child population; $38 \%$ of Australia's Aboriginal and Torres 
Strait Islander population live in major cities. ${ }^{12}$ Brisbane has the highest number of Aboriginal and Torres Strait Islander residents of all Australian capital cities. ${ }^{12}$ Despite the large population size, little is known about the health service utilisation patterns of Aboriginal and/or Torres Strait Islander children in urban settings. Such data are arguably important to understand the context of health outcomes and future planning of appropriate health services. Thus, our overall aim was to describe health service utilisation data in an urban Aboriginal and/or Torres Strait Islander setting. Specifically, our primary objective was to determine the incidence of health-care attendance amongst a cohort of urban Aboriginal and/or Torres Strait Islander children aged $<5$ years over a 12 -month period. Our secondary objectives were to describe the type of services accessed, the reasons for choice of health service provider and the primary reasons for presentation to those services.

\section{Methods \\ Design}

We undertook an analysis of health service utilisation data collected at baseline and during 12 months of follow-up in a prospective cohort study of acute respiratory illnesses (ARI) in children aged $<5$ years registered with an Aboriginal-owned and operated primary health-care service. The full study protocol has been previously published. ${ }^{13}$ This article presents data for the enrolled Aboriginal and/or Torres Strait Islander children.

\section{Setting and recruitment}

This study was undertaken at Caboolture Community Medical (CCM), an Aboriginal-owned and operated, comprehensive primary health-care service in Caboolture, Qld., Australia. Although CCM focuses on Indigenous patients, it provides services to any person in the district, irrespective of Indigenous status. The clinic is located within the Moreton Bay Regional Council (MBRC), with a resident population of 378045 in 2011, of whom $2.24 \%$ identified as Aboriginal and/or Torres Strait Islander (MBRC, 2017). In addition to CCM, there are five Aboriginal Community Controlled Health Service clinics in the MBRC region. Since its opening in 2011, CCM has provided services to 11229 clients, of whom 59\% identify as Aboriginal and/or Torres Strait Islander, equating to approximately $78 \%$ of the MBRC Aboriginal and/or Torres Strait Islander population. The clinic is predominantly staffed by Aboriginal and Torres Strait Islander professionals. The clinic is situated in the Caboolture central business district, within a 5-min walk of a train station and regular bus services, and it provides free parking. It also operates a client transport service for those with difficulty accessing the clinic.

Parents or guardians of children attending CCM for any reason, including accompanying another person, were approached by an Aboriginal PhD candidate and/or Aboriginal research officer at the time of presentation to determine whether they would like to participate in the cohort study. Children were eligible for inclusion in the cohort study if they were aged $<5$ years at enrolment, registered as a patient at CCM and if the parent or guardian was willing and able to complete study requirements. Children were excluded if the family was planning to move from the study area in the next 12 months. Written informed consent was obtained following provision of plain language statement. For this analysis, data from children in the cohort study were included if the child was identified as Aboriginal and/or Torres Strait Islander and had completed active follow-up in the cohort study.

\section{Ethical considerations}

The study was approved by Queensland Children's Health Services Human Research Ethics Committee (HREC/12/QRCH/169), the Medical Research Ethics Committee of the University of Queensland (2012001395) and the Human Research Ethics Committee of the Queensland University of Technology (1300000741). The study was registered with the Australian New Zealand Clinical Trials Registry (ACTRN 12614001214628). Cultural oversight was provided by an Aboriginal and/or Torres Strait Islander Research Reference Group.

\section{Data collection}

Data were collected at enrolment and monthly for 12 months following enrolment via detailed questionnaires administered by an Aboriginal research officer/PhD candidate to parents/carers and through a review of medical records at CCM. Data collected included demographic, epidemiological and clinical data at baseline and at each monthly follow-up. Health service utilisation data collected at each monthly time point included: the type of health provider used, the reason for presentation, the number of presentations over the previous month and hospital admissions. For the purpose of this analysis, ARI was defined an illness with cough and presence of one or more other local or systemic symptoms (e.g. runny nose, fever, shortness of breath, wheeze). Children were withdrawn if parents/carers withdrew consent at any time during the study or if monthly contacts were unsuccessful for two consecutive months, at which time children were considered lost to follow-up (LFTU).

\section{Data analyses}

Data analysis and management were under taken using Stata 13.1 software (Stata Corp, College Station, TX, USA). Descriptive analyses were performed. Health service utilisation rates, overall and by service provider and reason for presentation, were calculated by dividing the number of encounters by the total child-months of observation and reported as incidence rates per 100 child-months with the corresponding 95\% confidence interval (CI). Months in which follow-up contacts were not successful were excluded from the denominator; hence, months of observation were calculated by subtracting the date of enrolment from the date of last study contact with the family and then excluding the total number of months in which contacts were not successful. Given the small sample size, imputation methods to account for missing data were not employed. Age-specific rates were calculated using the child's age at the time he or she presented to the health service provider rather than age at time of enrolment. Backwards step-wise logistic regression was undertaken to compare selected characteristics between children who were and were not withdrawn during the follow-up period. 


\section{Results}

Between February 2013 and October 2015, 403 children were approached for participation in the cohort study, and 200 children were enrolled. Of those not enrolled, 72 (35.4\%) refused, $41(21.2 \%)$ were ineligible, and $88(43.3 \%)$ were not enrolled for other reasons; 73 parents wanted to think about it but did not contact research staff again, and for the remainder, the child was not with a parent/legal guardian, and hence, consent could not be obtained. Twenty children were excluded from this analysis as they identified as non-Aboriginal and/or Torres Strait Islander. There were no significant differences in age and gender between those enrolled and not enrolled (data not shown); no other characteristics of children who were not enrolled were collected.

Of the 180 Aboriginal and/or Torres Strait Islander children, the median age at enrolment was 18.4 months (interquartile range (IQR) 7.72-34.8), and 51\% were male; 1541 child-months of observation were available for analysis (mean 7.7 months per child, $95 \%$ CI $7.1-8.3)$. In total, $10 \%(n=18)$ of children had a known chronic condition. The detailed demographic, household, environmental and cultural characteristics of the children in the cohort study are presented in Table S1, Supporting Information. Key socio-economic indicators included the following: $76.5 \%$ of children lived in households with a total annual income of < $\$ 52$ 000; $41 \%$ were in single-parent households; $30.1 \%$ attended childcare; and $72 \%$ were regularly exposed to environmental tobacco smoke.

In total, 56 children were withdrawn during the study (31.1\%); parents/carers withdrew consent for 10 children, and the remainder were LTFU. The mean months of observation for children who were withdrawn were 2.5 (95\% CI 1.9-3.2) and 10.1 months (95\% CI 9.8-10.4) for children who were not withdrawn. There were no differences in age, gender, care type at home (e.g. single parent, both parents), household income, number of siblings, father's employment status, mother's education and presence of chronic illness between children withdrawn and those who were not. Children who were withdrawn were more likely to have fathers who did not finish high school (odd ratio (OR): 4.2, 95\% CI 1.9-9.5), or their father's educational status was unknown (OR 5.1, 95\% CI 1.8-14.7), compared to those not withdrawn.

In total, 783 health service encounters with any provider were recorded during the follow-up period for an overall incidence of 51 encounters per 100 child-months of observation $(95 \% \mathrm{CI}$ 48.7-56.5). All children accessed a health service provider at least once during their period of follow-up, and 15 children $(6 \%)$ had 10 or more encounters. Health service utilisation rates per $100-$ child-months were the highest in children aged 1 to $<2$ years (64.6, 95\% CI 58.0-73.6) (Table 1). Presentation rates per 100 child-months were the highest for ARI $(30.0,95 \%$ CI $27.8-33.9)$ and other illnesses $(12.5,95 \%$ CI $11.5-15.5)$ (Table 2). The majority of these illnesses $(83 \%)$ were for common childhood conditions such as otitis media $(23 \%)$, gastrointestinal disturbances $(9 \%)$, skin infections $(12 \%)$, throat infections $(8 \%)$, rashes $(11 \%)$ and minor injuries $(9 \%)$. Presentation rates per 100 child-months to specific services were the highest for CCM $(31.9,95 \%$ CI 30.5-36.8), followed by other mainstream GPs (8.0, 95\% CI 6.8-10.0) (Table 2).
At enrolment, 169 of 180 (94\%) parents/carers reported that CCM was their child's usual health-care provider. With respect to the reasons for choosing CCM as the usual primary health-care provider for their child, $77 \%$ of parents reported they considered CCM Aboriginal and/or Torres Strait Islander friendly, 79\% because CCM bulk bills, $41 \%$ because it was close to home and $18 \%$ because they liked the doctor.

\section{Discussion}

Whilst over a third of Aboriginal and/or Torres Strait Islander children live in urban areas, there are limited data on their health-care utilisation patterns. We examined health-care utilisation over a period of up to 12 months in young urban Aboriginal and/or Torres Strait Islander children with substantial socioeconomic disadvantage. In this cohort, health-care utilisation was frequent across all yearly age groups, with ARI the most common reason for presentation. CCM was the primary source of health care, followed by mainstream GPs, with other Aboriginal Medical Services and Emergency Departments (EDs) accessed infrequently when the child required care.

There are limited studies that are directly comparable with our results and none with recent data. Furthermore, none have reported incidence rates based on exact measures of child-months of observation. Most studies analysing health service use in Aboriginal and/or Torres Strait Islander children have analysed data from remote Aboriginal and/or Torres Strait Islander communities or hospital ED data. Data from a decade of GP surveys ${ }^{14}$ suggested that only $1.7 \%$ of patients visiting GPs identified as being of Aboriginal and/or Torres Strait Islander origin. Aboriginal and/or Torres Strait Islander Australians access mainstream GP services at a similar rate (5630 per 1000 population) as nonAboriginal and/or Torres Strait Islander Australians (5550 per 1000 population), although these data represented all ages. ${ }^{15} \mathrm{Ou}$ et al. ${ }^{9}$ analysed 2004 data for children (0-12 months of age) from the Longitudinal Study for Australian Children (LSAC) and found that Aboriginal and/or Torres Strait Islander infants $(\underline{n}=154)$ in the first 12 months of life had a lower mean number of visits to any service than non- Aboriginal and/or Torres Strait Islander infants $(n=3746)$ (2.5 vs. 3.1, respectively, $P<0.001) .{ }^{9}$ It is not possible to directly compare these findings with our study given the different denominators used and the differing duration of follow-up for individual children in the two studies. Furthermore, the limitations of the LSAC study ${ }^{9}$ are the small number of Aboriginal and/or Torres Strait Islander children included, the clustered sample design of postcodes that excluded remote postcodes, postcodes on Aboriginal and/or Torres Strait Islander lands and postcodes that had more than 50\% Aboriginal and/or Torres Strait Islander children. Furthermore, the data from the studies mentioned above are now relatively old, and there has been substantial growth in Aboriginal and/or Torres Strait Islander-specific primary care services in the following decade.

In total, $94 \%$ of parents stated that CCM was the usual health service they utilise when their child is ill. Having a regular GP allows for continuity of care, which leads to improved care and better health outcomes. ${ }^{16,17}$ Parents/Carers reported that their major reasons were that it was Aboriginal and/or Torres Strait Islander friendly (i.e. predominantly Aboriginal and/or Torres Strait Islander staff and provided a range of Aboriginal and/or 


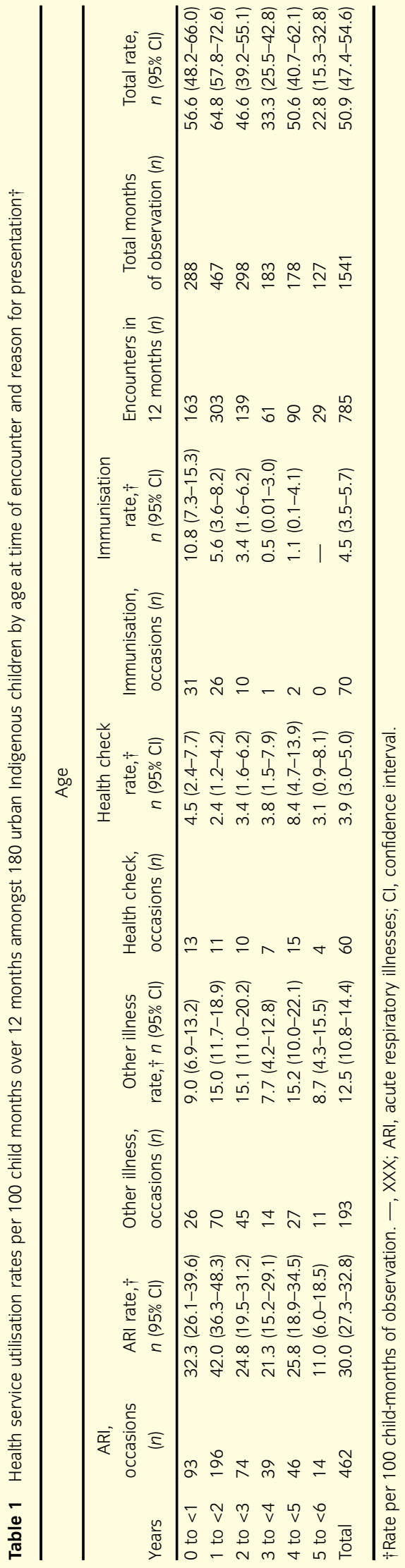

Torres Strait Islander specific services) and/or offered bulk billing, the latter likely to be important given the low socio-economic profile of families in the study. ${ }^{18}$ Similarly, that it was also close to home for $41 \%$ of families and, given its location, is readily accessible by public transport is likely to influence decisions to use the service. It is well established that culturally aware services are critical to improving the Aboriginal and/or Torres Strait Islander health gap in Australia. ${ }^{19,20}$ Such services also facilitate and encourage health service use. The use of mainstream GPs and EDs as alternatives relative to the low reported use of alternative Aboriginal Medical Services in the region potentially reflects the times in which health care is needed (e.g. after-hours services such as home doctors and services open on weekends given CCM is not) or an illness where parents considered taking the child to an ED.

A number of studies have identified that ARIs are the most common reason for presentation to primary health-care services amongst Aboriginal and/or Torres Strait Islander children. ${ }^{1,14,21}$ Similarly, ARIs were the most common reason for presentation in this study $(58 \%)$, suggesting that they are likely the predominant cause of health service utilisation irrespective of urban or remote residence. The ARI rate is not surprising given that respiratory illnesses are the second most common acute cause of hospitalisation amongst Aboriginal and/or Torres Strait Islander Australians and the most common self-reported chronic disease amongst Aboriginal and/or Torres Strait Islander people aged $<34$ years. ${ }^{22}$ Illnesses reported in the 'other' category were similar to those seen in general practices in Australia ${ }^{14}$ : fevers, ear infections, rashes, viral infections, minor injuries and skin infections, and few visits were for pre-existing chronic conditions other than asthma or eczema.

The strengths of this study are that Aboriginal researchers and service providers were actively engaged in the design of the study and undertook all recruitment, data collection and follow-up procedures. This facilitated family engagement and provided safety around the collection of sensitive information. Given that this was a single-centre study, the major limitation is that the profile of children presenting to CCM may differ from other urban Aboriginal and Torres Strait Islander communities, and there are likely to be differences between children who were and were not enrolled. This would be important if those who did not enrol did not access services frequently. Similarly, the ARI rates observed may be a reflection of the focus of the overall primary study, which was ARI, and the rates of presentations to health care for ARI may differ in children not enrolled. ${ }^{13}$ These limitations are similar to most studies of Aboriginal and/or Torres Strait Islander health that have been conducted in limited geographical regions, and ongoing caution is required with respect to generalising the findings from those studies, including this study, across Australia. These limitations support the need for larger studies in multiple different communities to determine if health-care utilisation is similar in other urban settings. Finally, approximately one third of children enrolled were withdrawn over the study period, and this results in uncertainty around utilisation rates. However, in the context of the complexity of this community and the socioeconomic profile of families involved, ${ }^{18}$ we considered overall retention to be good and to be a reflection of the importance of Aboriginal researchers undertaking the work. The reason why the educational status of fathers was a predictor of withdrawal is 


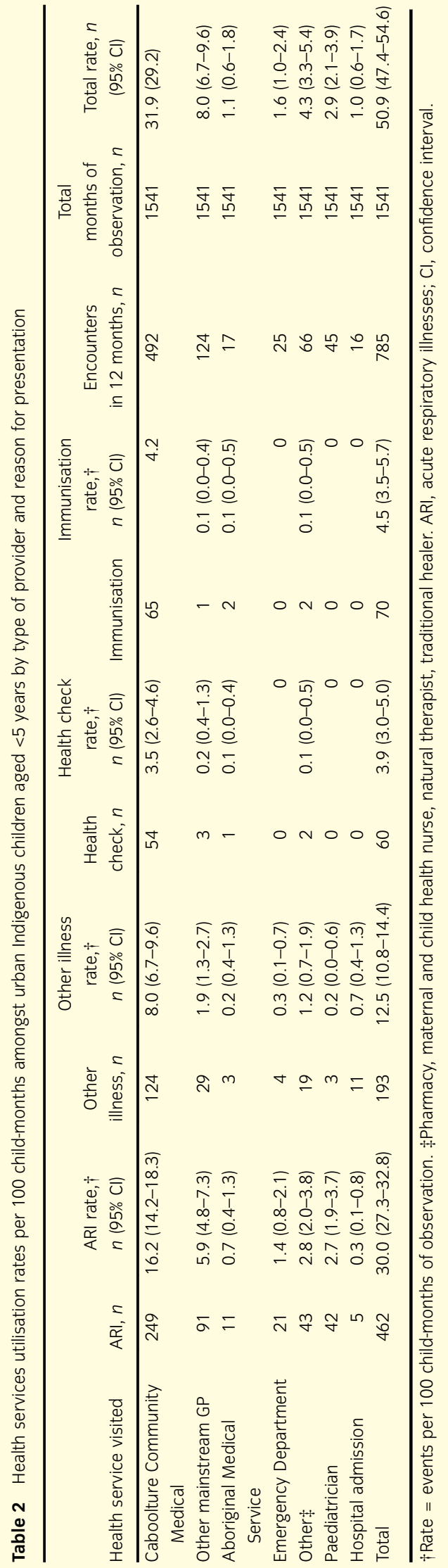

difficult to explain and warrants further investigation in future studies. Despite the limitations, this study provides some important insights into the current health-seeking practices in some urban Aboriginal and Torres Strait Islander communities. If access to services is no longer a barrier to seeking health care, this would be a positive outcome of the efforts across Australia to reduce these barriers. However, access to care does not necessarily translate to satisfaction with care or the quality of the care received, and further studies are required in the primary care setting to address those issues.

We aimed to describe health service utilisation data amongst a cohort of urban Aboriginal and/or Torres Strait Islander children aged $<5$ years over a 12 -month period. We identified a cohort of families who have a usual primary health-care provider and are accessing primary health services for both illnesses and well-child services, and we confirmed ARI as a predominant cause of health service use. Hence, in this population and setting, there are several opportunities to address potential health risks and to provide health education and intervention programmes.

\section{Acknowledgements}

This study would not have been possible without the cooperation and collaboration provided by the staff of Caboolture Community Medical, Caboolture, as well as the participation of the study families. We thank Melissa Dunbar for her assistance with the recruitment and follow-up of study children and Jack Roberts for his assistance with data cleaning. Funding for the study was provided through a Queensland Children's Medical Research Institute project grant, a University of Queensland Foundation Research Excellence Award, a Queensland Government Smart Futures Fellowship (51008) and a QUT Indigenous Health startup research grant to KF O'Grady. KF O'Grady is funded by a National Health $\delta$ Medical Research Council (NHMRC) Career Development Fellowship (1045147). KK Hall is funded by an Australian Postgraduate Award (2014-2016) and a supervisor top-up scholarship through the Centre for Research Excellence for Lung Health in Aboriginal and Torres Strait Islander children. $\mathrm{AB}$ Chang is funded by a NHMRC Practitioner Fellowship (1058213).

\section{References}

1 Kearns T, Clucas D, Connors C, Currie BJ, Carapetis JR, Andrews RM. Clinic attendances during the first 12 months of life for Aboriginal children in five remote communities of northern Australia. PLOS One 2013; 8: e58231.

2 Australian Institute of Health and Welfare. Australia's Health 2014. Canberra: The Institute; 2014.

3 Waterworth P, Rosenberg M, Braham R, Pescud M, Dimmock J. The effect of social support on the health of Indigenous Australians in a metropolitan community. Soc. Sci. Med. 2014; 119C: 139-46.

4 Pink B, Allbon P. The Health and Welfare of Australia's Aboriginal and Torres Strait Islander Peoples. Canberra: Commonwealth of Australia; 2008.

5 Davy C, Harfield S, McArthur A, Munn Z, Brown A. Access to primary health care services for Indigenous peoples: A framework synthesis. Int. J. Equity Health 2016; 15: 163.

6 Scrimgeour MJ, Scrimgeour D. Health Care Access for Aboriginal and Torres Strait Islander People Living in Urban Areas, and Related 
Research Issues: A Review of the Literature. Discussion Paper Series, 1-80. Darwin: Cooperative Research Centre for Aboriginal Health; 2007.

7 Ou L, Chen J, Garrett P, Hillman K. Ethnic and Indigenous access to early childhood healthcare services in Australia: Parents' perceived unmet needs and related barriers. Aust. N. Z. J. Public Health 2011; 35: 30-7.

8 Anderson I, Crengle S, Leialoha Kamaka M, Chen TH, Palafox N, Jackson-Pulver L. Indigenous health in Australia, New Zealand, and the Pacific. Lancet 2006; 367: 1775-85.

9 Ou L, Chen J, Hillman K, Eastwood J. The comparison of health status and health services utilisation between Indigenous and nonIndigenous infants in Australia. Aust. N. Z. J. Public Health 2010; 34: 50-6.

10 Szilagyi PG. Managed care for children: Effect on access to care and utilization of health services. Future Child. 1998; 8: 39-59.

11 Bissar AR, Oikonomou C, Koch MJ, Schulte AG. Dental health, received care, and treatment needs in 11-to 13-year-old children with immigrant background in Heidelberg, Germany. Int. J. Paediatr. Dent. 2007; 17: 364-70.

12 Australian Bureau of Statistics. The Health and Welfare of Australia's Aboriginal and Torres Strait Islander Peoples 2010. Canberra: Australian Government; 2011. Available from: http://www.abs.gov.au/ AUSSTATS/abs@.nsf/lookup/4704.0Chapter2500ct+20102016.

13 Hall KK, Chang AB, Sloots TP et al. The respiratory health of urban indigenous children aged less than 5 years: Study protocol for a prospective cohort study. BMC Pediatr. 2015; 15: 56-64.

14 Britt HMGC, Henderson J, Bayram C et al. General Practice Activity in Australia 2014-15. Sydney: University of Sydney; 2015.

15 Australian Institute of Health and Welfare. Access to Health Services for Aboriginal and Torres Strait Islander People. Canberra: The Institute; 2011.

16 Turbitt E, Freed GL. Regular source of primary care and emergency department use of children in Victoria. J. Paediatr. Child Health 2015; 52: 303-7.
17 Haggerty J, Reid RJ, Freeman G, Starfield BH, Adair CE, McKendry R. Continuity of care: A multidisciplinary review. BMJ 2003; 327: 1219-21.

18 Hall KKCA, Anderson J, Dunbar M, Arnold D, O'Grady KF. The characteristics and respiratory risk profile of children aged less than 5 years presenting to an urban, Indigenous friendly, comprehensive primary health practice in Australia. J. Paediatr. Child Health 2017.

19 Johanson RP, Hill P. Indigenous health: A role for private general practice. Aust. Fam. Physician 2011; 40: 16-9.

20 Bath J, Wakerman J. Impact of community participation in primary health care: What is the evidence? Aust. J. Prim. Health 2015; 21: 2-8.

21 Clucas DB, Carville KS, Connors C, Currie BJ, Carapetis JR, Andrews RM. Disease burden and health-care clinic attendances for young children in remote aboriginal communities of northern Australia. Bull. World Health Organ. 2008; 86: 275-81.

22 Australian Institute of Health and Welfare. The Health and Welfare of Australia's Aboriginal and Torres Strait Islander Peoples: 2015. Canberra: The Institute; 2015.

\section{Supporting Information}

Additional Supporting Information may be found in the online version of this article at the publisher's web-site:

Table S1. Characteristics of 180 urban Aboriginal and/or Torres Strait Islander children enrolled in a prospective cohort study of acute respiratory illness. 